\title{
Front Matter: Volume 11197
}

, "Front Matter: Volume 11197," Proc. SPIE 11197, SPIE Future Sensing Technologies, 1119701 (13 December 2019); doi: 10.1117/12.2559573

SPIE. Event: SPIE Future Sensing Technologies, 2019, Tokyo, Japan 


\title{
PROCEEDINGS OF SPIE
}

\section{SPIE Future Sensing Technologies}

\author{
Masafumi Kimata \\ Christopher R. Valenta
}

Editors

14 November 2019

Tokyo, Japan

Sponsored and Published by

SPIE 
The papers in this volume were part of the technical conference cited on the cover and title page. Papers were selected and subject to review by the editors and conference program committee. Some conference presentations may not be available for publication. Additional papers and presentation recordings may be available online in the SPIE Digital Library at SPIEDigitalLibrary.org.

The papers reflect the work and thoughts of the authors and are published herein as submitted. The publisher is not responsible for the validity of the information or for any outcomes resulting from reliance thereon.

Please use the following format to cite material from these proceedings:

Author(s), "Title of Paper," in SPIE Future Sensing Technologies, edited by Masafumi Kimata, Christopher R. Valenta, Proceedings of SPIE Vol. 11197 (SPIE, Bellingham, WA, 2019) Seven-digit Article CID Number.

ISSN: 0277-786X

ISSN: 1996-756X (electronic)

ISBN: 9781510631113

ISBN: 9781510631120 (electronic)

Published by

SPIE

P.O. Box 10, Bellingham, Washington 98227-0010 USA

Telephone +1 3606763290 (Pacific Time) · Fax +1 3606471445

SPIE.org

Copyright (c) 2019, Society of Photo-Optical Instrumentation Engineers.

Copying of material in this book for internal or personal use, or for the internal or personal use of specific clients, beyond the fair use provisions granted by the U.S. Copyright Law is authorized by SPIE subject to payment of copying fees. The Transactional Reporting Service base fee for this volume is $\$ 21.00$ per article (or portion thereof), which should be paid directly to the Copyright Clearance Center (CCC), 222 Rosewood Drive, Danvers, MA 01923. Payment may also be made electronically through CCC Online at copyright.com. Other copying for republication, resale, advertising or promotion, or any form of systematic or multiple reproduction of any material in this book is prohibited except with permission in writing from the publisher. The CCC fee code is 0277$786 \mathrm{X} / 19 / \$ 21.00$.

Printed in the United States of America by Curran Associates, Inc., under license from SPIE.

Publication of record for individual papers is online in the SPIE Digital Library.

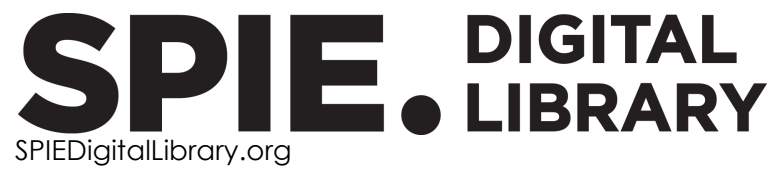

Paper Numbering: Proceedings of SPIE follow an e-First publication model. A unique citation identifier (CID) number is assigned to each article at the time of publication. Utilization of CIDs allows articles to be fully citable as soon as they are published online, and connects the same identifier to all online and print versions of the publication. SPIE uses a seven-digit CID article numbering system structured as follows:

- The first five digits correspond to the SPIE volume number.

- The last two digits indicate publication order within the volume using a Base 36 numbering system employing both numerals and letters. These two-number sets start with $00,01,02,03,04$, $05,06,07,08,09,0 A, 0 B \ldots$ OZ, followed by 10-1Z, 20-2Z, etc. The CID Number appears on each page of the manuscript. 


\title{
Contents
}

\author{
vii Authors \\ ix Conference Committee
}

\section{SESSION $1 \quad$ FUTURE SENSING TECHNOLOGIES I}

1119704 Performance evaluation of the optical navigation electronics of HAYABUSA2 (Invited Paper) [11197-3]

\section{SESSION 2 FUTURE SENSING TECHNOLOGIES II}

1119706 Plastic waste monitoring and recycling by hyperspectral imaging technology (Invited Paper) [11197-5]

1119708 Waveguide AR HUDs: a critical enabler for autonomous vehicles (Invited Paper) [1 11 197-7]

$1119709 \quad$ Multimodal imaging based digital holography (Invited Paper) [11197-8]

\section{POSTER SESSION}

11197 OA Using transfer learning technique for SAR automatic target recognition [11197-9]

11197 OB Research on submarine terrain reconstruction technology based on laser polarization characteristics [11197-10]

11197 OC Multipath angle estimation for low-flying target of multi-frequency shipborne radar [1 1 197-11]

11197 OE Planar optical sensor for trichlorofluoromethane [11197-13]

11197 OF Effect of windowing on a sensing signal generated by self-mixing interferometry [11197-14]

$11197 \mathrm{OH} \quad$ Recovery of defocused 3D data for focusing-optics-based amplitude-modulated continuouswave laser scanner [1 11 197-18]

11197 Ol Achieving high resolution measurement using laser diode operating at period one [11197-19]

11197 OK An absolute linear displacement sensor [11197-21]

11197 OL Study on guide star selection for infrared star sensors [1 11 197-22] 
11197 OM Multi-band optical imaging sensor for coastal ocean color remote sensing [11197-23]

11197 ON Recovery of phase modulation via residual neural network [11197-24]

$1119700 \quad$ Automatic organic light-emitting diode display Mura detection model based on human visual perception and multi-resolution [111197-25]

11197 OP Fiber-interferometer-based dynamic displacement sensor with triangle and stepwise phase modulation on the reference light [1 $11197-26]$

$111970 Q \quad$ Enhanced seismic sensing based on epoxy waveguide Bragg gratings [11197-27]

11197 OR High-speed quantum cascade detector with frequency response of over 20 GHz [11197-28]

11197 OS Accessing refractive errors via eccentric infrared photorefraction based on deep learning [1 11 197-29]

11197 OT Distributed matrix methods of compression, masking and noise-resistant image encoding in a high-speed network of information exchange, information processing and aggregation [11197-30]

$111970 \mathrm{U}$ Autonomous steel casting recognition and positioning on the unmanned automatic production line based on binocular vision [11197-31]

11197 OW Adaptive CA-CFAR thresholding based on an optimal number of reference cells [11197-34]

$111970 X \quad$ Features of information processing in the onboard two-position small-sized radar based on UAVs [1 $1197-35]$

11197 OY Short-range supercontinuum based lidar for combustion diagnostics [11197-36]

$111970 Z$ Image fusion with guided saliency [11197-37]

1119710 Microplastics characterization by hyperspectral imaging in the SWIR range [11197-38]

1119711 A simple dual-frequency noise laser rangefinder [11197-39]

1119712 Utilizing full neuronal states for adversarial robustness [1 $11197-40]$

1119713 Single detector polarization lidar for atmospheric studies [1 11 197-41]

$1119715 \quad$ Ultra-rapid large deployable antennas using UAV for HF and lower frequency applications [11197-43]

1119716 Stability of multiwavelength fiber laser employing semiconductor optical amplifier in nonlinear optical loop mirror with polarization maintaining fiber [1 $1197-44]$

1119717 Breast surface variation phase map analysis with digital fringe projection [11197-45] 
1119718 Output characteristics of polarization-maintaining fiber ring resonator: model and experiment [11197-46]

1119719 Spectrum selection for imaging polarization sensor [11197-47]

$111971 \mathrm{~A}$ Sensor system for measuring temperature changes using alternative detection of polarization changes [11197-48]

11197 1B Automatic target tracking with time-delayed measurements for unmanned surface vehicles [11197-49]

11197 IC Man-made object separation using polarimetric imagery [1 $1197-50]$ 
Proc. of SPIE Vol. $111971119701-6$

Downloaded From: https://www.spiedigitallibrary.org/conference-proceedings-of-spie on 26 Apr 2023 Terms of Use: https://www.spiedigitallibrary.org/terms-of-use 


\section{Authors}

Numbers in the index correspond to the last two digits of the seven-digit citation identifier (CID) article numbering system used in Proceedings of SPIE. The first five digits reflect the volume number. Base 36 numbering is employed for the last two digits and indicates the order of articles within the volume. Numbers start with 00, 01, 02, 03, 04, 05, 06, 07, 08, 09, 0A, 0B...0Z, followed by 10-1Z, 20-2Z, etc.

Abed AL-Asadi, Hamid Ali, 16

Ahmad Hambali, N. A. M., 16

Al Hadhrami, Esra, OA

Al Mufti, Maha, OA

Ali, N., 16

Aris, H., 17

Awatsuji, Yasuhiro, 09

Ayub, Rafiq, 13

Azaña, Josè, 11

Azmi, M. A. M., 17

Bonifazi, Giuseppe, 10

Bott, Patrick, $\mathrm{OQ}$

Burylev, Dmitry A., OT, OX

Chen, $\mathrm{XU}, \mathrm{OB}$

Chen, Zhuqiu, 0

Cheng, Chu-Ming, OS

Cho, Ming-Hsiang, 15

Chun, Joohwan, OC

Dougakiuchi, Tatsuo, OR

Edamura, Tadataka, OR

Fiore, Ludovica, 10

Gain, Alex, 12

Gao, Hongbiao, ow

Genty, Goery, OY

Girschikofsky, Maiko, OE, OQ

Grant, Alastair J., 08

Grenar, David, 1A

Guo, Qi, 19

Guo, Qinghua, OF, Ol

Han, Jungwook, 1B

Hayashi, Neisei, $\mathrm{OH}$

Hellmann, Ralf, $\mathrm{OE}, \mathrm{OQ}$

Hessler, Steffen, OE, OQ

Hihara, Hiroki, 04

Hsieh, Po-Yuan, 00

Hu, Wen-Ying, 15

Hu, Zongfu, 18

Husshini, N. F. H., 16

Islam, Md Nazrul, 1C

Jiang, Zhiguo, 18

Jiang, Zhiping, OW

Jin, Lei, $\mathrm{OH}$

Jung, Jihyun, OC

Kapranova, Ekaterina A., OT

Kashiwada, Shosaku, 10

Katsumata, Takaaki, OM

Kefer, Stefan, $0 Q$

Kim, Jinuk, OC

Kim, Jinwhan, 1B
Kryachko, Alexander F., OX

Kumar, Manoj, 09

Kyselak, Martin, $1 \mathrm{~A}$

Lee, Joohyun, OC

Li, Jie-En, ON, 0O, OS

$\mathrm{Li}$, Xudong, 19

$\mathrm{Li}$, Yefei, OB

Lin, Ming-Yung, 15

Lin, Shu-Yu, 15

Liu, Lei, OB

Liv, Sifan, $\mathrm{OH}$

Liu, Yang, OK

Massoud, Ali, OW

Masuda, Tetsuya, 04

Matoba, Osamu, 09

Mou, Chuan, OK

Nenashev, Sergey A., OT

Nenashev, Vadim A., OT, OX

Nie, Bairun, 01

Niki, Masato, OM

Norhaimi, W. M. W., 17

Ogawa, Naoko, 04

Oh, Yougeun, OC

Ohashi, Yuta, OP

Oishi, Tomohiko, OM

Okada, Tatsuaki, 04

Onori, Daniel, 11

Ootake, Hisashi, 04

Peng, LingLi, OK

Pickering, Mark, $1 \mathrm{C}$

Popovich, Milan M., 08

Quan, Xiangyu, 09

Rajput, Sudheesh K., 09

Retnasamy, $V_{\text {., }} 17$

Ruan, Yuxi, OF, Ol

Ryczkowski, Piotr, OY

Ryvlin, Dimitrij, OE

Saleh, Abba, OY

Sano, Junpei, 04

Sauli, Z., 17

SchmauB, Bernhard, $O Q$

Sergeev, Alexander M., OT

Serranti, Silvia, 06, 10

Set, Sze Yun, $\mathrm{OH}$

Shahimin, M. M., 16, 17

Sharukh, Mohammed, 13

Shepeta, Alexander P., OX

Siegelmann, Hava T., 12

Slavicek, Karel, 1A 
Son, Nam-sun, 1B

Song, Jiaxiao, $O B$

Su, Jian-Jia, ON, 0O, OS

Su, Riliang, OW

Suh, Jinbae, OC

Sun, Jwo-Shiun, 15

Taha, Bilal, OA

Tahtali, Murat, $1 \mathrm{C}$

Takemae, Shinya, OP

Takeshima, Ayaka, 10

Takeuchi, Hisato, 10

Tan, Hiroyuki, OM

Tanaka, Akihiko, OM

Tanaka, Yosuke, OP

Tien, Chung-Hao, ON, 0O, OS

Toivonen, Juha, OY

Tong, Jun, OF, Ol

Tseng, Jin-Shing, OS

Tsuda, Yuichi, 04

Vairavan, R., 17

Wahid, M. H. A., 16

Waldern, Jonathan D., 08

Waldvogel, Siegfried R., OE

Wang, Han, OF

Wang, Hong, OW

Werghi, Naoufel, OA

Wong, K., 17

Wu, Feng, OL, OU, OZ

Wu, Liang, OK

$\mathrm{Xi}$, Jiangtao, OF, Ol

Xiang, Fulin, $\mathrm{OH}$

Xiang, Ruxi, OL, OU, OZ

$\mathrm{XU}$, Qingquan, $\mathrm{OL}, \mathrm{OU}, \mathrm{OZ}$

Yamashita, Shinji, $\mathrm{OH}$

Yang, Chia-Chi, OS

Yang, Xiu, OL, OU

Yao, Yun-Zhen, ON

Yasin, M. N. M., 16

Yellapragada, Bhavani Kumar, 13

YU, Yanguang, OF, OI

Zeng, Li, OW

Zhang, $\mathrm{Chao}, \mathrm{OH}$

Zhang, Hao, 19

Zhang, Ying, 19

Zhang, Zheyuan, $\mathrm{OH}$

Zhao, Huijie, 19

Zhong, ZiQiang, OK

Zhou, Jie, OL, OU

Zhou, Yilun, OW

Zhu, Xifang, $\mathrm{OZ}$

Zhu, Zhi-Yu, ON, 0O, OS 


\title{
Conference Committee
}

\author{
Conference Chairs
}

Masafumi Kimata, Ritsumeikan University (Japan)

Christopher R. Valenta, Georgia Tech Research Institute (United States)

International Program Committee

Junichi Kudo, ATLA, Japan MOD (Japan)

John M. Pellegrino, Georgia Institute of Technology (United States)

Joseph A. Shaw, Montana State University (United States)

Hiroshi Shikata, Sumitomo Electric (Japan)

Conference Program Committee

Junichi Kudo, ATLA, Japan MOD (Japan)

John M. Pellegrino, Georgia Institute of Technology (United States)

Joseph A. Shaw, Montana State University (United States) 
Proc. of SPIE Vol. 11197 1119701-10

Downloaded From: https://www.spiedigitallibrary.org/conference-proceedings-of-spie on 26 Apr 2023 Terms of Use: https://www.spiedigitallibrary.org/terms-of-use 